\section{Efficient Cloning of SAGE Tags by Blunt-End Ligation of Polished Concatemers}

\author{
BioTechniques 34:692-694 (April 2003)
}

The serial analysis of gene expression (SAGE) has become an important technique for high-throughput transcriptome analysis. Since its first description in 1995 (1), SAGE has provided both quantitative and qualitative information concerning global gene expression in a variety of biological systems. Thereby, it has deepened our knowledge concerning developmental processes as well as pathogenic mechanisms (2-4). Furthermore, SAGE was shown to be a useful tool for genome annotation (5).

The common application of SAGE, however, was delayed and is still hampered by a technically demanding protocol. Recently, improvements have been made toward a more widespread use of this method (6-9). Several of the methodical advances concerned the cloning of the concatemers, as this was shown to be a crucial yet inefficient step. One protocol described the removal of biotinylated linkers by streptavidin-coated magnetic beads before cloning (10). The introduction of a heating step before separation of the concatemers by gel electrophoresis resulted in a better correlation between the migration distance in the gel and the concatemer length (11). This allowed the cloning of concatemers with, on average, significantly greater length. None of these protocols, however, has addressed the major problem underlying inefficient cloning of any given concatemer, which is the problem of "corrupted concatemer termini". Corrupted concatemer termini do not contain the nucleotide termini expected from normal endonuclease cleavage; consequently, concatenation has an inherent tendency to stop at these termini, as they prevent further elongation. Corrupted termini can arise from exonuclease activity present during/after restriction endonuclease cleavage, or from star activity of the restriction endonuclease used. These corrupted termini result in poor cloning efficiency of the concatemers, as these are subsequently cloned into a vector cleaved with a restriction endonuclease that yields cohesive ends compatible only with intact termini.

One approach to overcome this problem involves polishing the concatemer termini, thus allowing bluntend ligation. Although blunt-end ligation is generally less efficient than cohesive end ligation, it can result in a higher efficiency under conditions of enriched corrupted cohesive termini. To test this hypothesis, we used the exonuclease activity of bacteriophage T4 DNA polymerase to convert the $3^{\prime}$-protruding concatemer termini to bluntend DNA, as the commonly used fill-in reaction by Klenow is prohibited by the presence of protruding $3^{\prime}$ ends (12).

Concatemers were purified from biotinylated linkers by streptavidin-coated magnetic beads (Dynal, Hamburg, Germany) (10), kept at $65^{\circ} \mathrm{C}$ for $5 \mathrm{~min}$ to break concatemer aggregates (11) and separated in a $1 \%$ agarose gel (universal agarose; Peqlab, Erlangen, Germany). Fractions of 300-500 bp (fraction I) and 500-1000 bp (fraction II) in length were cut out and eluted from the gel matrix with the E.Z.N.A. Gel Extraction Kit (Peqlab). To compare blunt-end ligation with the established protocol of cloning concatemers into the $S p h$ I restriction site, each fraction was divided into two aliquots of $15 \mu \mathrm{L}$. To one aliquot, 1.2 U T4 DNA polymerase (New England Biolabs, Frankfurt, Germany) were added in the presence of $100 \mu \mathrm{M}$ each dNTP, and the reaction was run for $15 \mathrm{~min}$ at $12^{\circ} \mathrm{C}$ in $1 \times$ reaction buffer in a total volume of $20 \mu \mathrm{L}$. The reaction was stopped by heat-denaturing the enzyme at $75^{\circ} \mathrm{C}$ for $20 \mathrm{~min}$. This yielded a polished concatemer sample ready for blunt-end ligation. The other aliquot was kept at $4^{\circ} \mathrm{C}$ during this procedure (unpolished concatemer sample).

To clone concatemers, a modified pBluescript ${ }^{\circledR}$ vector (Stratagene, Amsterdam, The Netherlands) was used that contained an additional SphI recognition site between the BamHI and the EcoRI recognition site. Five micrograms of vector were cleaved either with 50 U SphI (New England Biolabs) or with 50 U Eco32I (MBI Fermentas, St. Leon-Rot, Germany) for $90 \mathrm{~min}$ at $37^{\circ} \mathrm{C}$ in a total volume of $100 \mu \mathrm{L}$. Eco32I represents an isoschizomer of EcoRV and was recently reported to improve blunt-end cloning by a decreased intrinsic single nucleotide deletion activity when compared to EcoRV (13). This should allow for more efficient blue/white selection. After cleavage by restriction endonuclease, vectors were dephosphorylated using $10 \mathrm{U}$ calf intestinal phosphatase (New England Biolabs), purified by agarose gel electrophoresis, and extracted using the same gel extraction kit as above (Peqlab). The entire unpolished samples were ligated into $100 \mathrm{ng} S p h \mathrm{I}$-cleaved pBluescript; the entire T4 DNA polymerase-polished samples were directly ligated after the heat denaturation step into $100 \mathrm{ng}$ Eco32I-cleaved pBluescript by adding $15 \mathrm{U}$ T4 DNA ligase (MBI Fermentas), $5 \mu \mathrm{L} 10 \times$ ligase buffer and water to a total volume of $50 \mu \mathrm{L}$. Ligations were carried out at $15^{\circ} \mathrm{C}$ for $19 \mathrm{~h}$. Thereafter, T4 DNA ligase was removed by StrataClean ${ }^{\mathrm{TM}}$ resin (Stratagene), and the ligation products were precipitated with ethanol in the presence of $70 \mu \mathrm{g}$ glycogen (Peqlab) and resuspended in $10 \mu \mathrm{L}$ water. Then, $1.5 \mu \mathrm{L}$ each ligation product was added to 50 $\mu \mathrm{L}$ ElectroTen-Blue ${ }^{\mathrm{TM}}$ electroporation competent cells $\left(\geq 1 \times 10^{10}\right.$ transformants/ $\mu g$; Stratagene). After electroporation at $1.8 \mathrm{kV}$ (E. coli pulser; Bio-Rad Laboratories $\mathrm{GmbH}$, München, Germany) in 0.2-cm-wide cuvettes (Peqlab), transformation mixtures were spread on 145-mm-wide LB agar plates containing $50 \mu \mathrm{g} / \mathrm{mL}$ ampicillin (Sigma, Taufkirchen, Germany) and the X-gal/IPTG system (Peqlab) for blue/white selection. Transformed cells were counted the next day. A substantial increase of transformants was observed for T4 DNA polymerase-treated concatemers when compared to the corresponding SphI ligation (Table 1). A 3.4-fold increase for fraction I and a 2fold increase for fraction II were observed. On all plates, less than $1 \%$ blue colonies were detected.

To check for insert length, the inserts of 40 randomly picked white clones from Eco32I ligations were amplified by colony-PCR and separated in a $1 \%$ agarose gel. Thirty-seven of 40 selected clones $(92.5 \%)$ from fraction I and 36 of 40 selected clones $(90 \%)$ from frac- 
Table 1. Cloning Efficiency

\begin{tabular}{|lccc|}
\hline Ligation & $\begin{array}{c}\text { Transformants/ } \\
\text { Reaction }\end{array}$ & $\begin{array}{c}\text { Total Number of } \\
\text { Transformants }\end{array}$ & \% Ligation \\
\hline Fraction I, Sphl & 7260 & 96800 & 100 \\
Fraction I, Eco32I & 25245 & 336600 & 347 \\
Fraction II, Sphl & 3960 & 52800 & 100 \\
Fraction II, Eco32I & 8085 & 107800 & 204 \\
pBluescript, Sphlc & 9 & n.d. & n.d. \\
pBluescript, Eco32|c & 330 & n.d. & n.d. \\
\hline
\end{tabular}

aCalculated number of transformants using the complete gel-eluted concatemers of a given size fraction.

bThe clone number of the corresponding Sph SAGE library was set to $100 \%$. cSelf ligation of restriction endonuclease-cleaved and dephosphorylated vector. n.d., not determined. tion II contained an insert, demonstrating the effective blue/white screening of an Eco32I blunt-ended vector (Figure 1). The average insert length was 292 and $545 \mathrm{bp}$ for fractions I and II, respectively. Similar insert lengths were found for SphI clones (313 and $525 \mathrm{bp}$, respectively), demonstrating that the inherent T4 DNA polymerase exonuclease activity did not affect the concatemer length. The rather short insert size was due to a steep decline of long concatemers in the fractions. Two of the clones without insert (marked by a filled square in Figure 1) were due to vector religation; the other ones yielded likely no PCR products because of corrupted/missing primer site(s), as a subsequent analysis revealed (data not shown). Taken together, the total number of insert containing clones that can be obtained amounts to more than 330000 for fraction I and 107000 for fraction II after blunt-end ligation (Table 1). Moreover, greater than or equal to $90 \%$ of them contain an insert. This exceeds for each ligation by far the clone numbers $\left(<3 \times 10^{3}\right)$ typically analyzed during a SAGE experiment.

To prove finally that the treatment with T4 DNA polymerase did not affect the quality of the concatemers, several clones were sequenced. Again, no difference between polished and unpolished concatemers was observed (data not shown).

These data demonstrate that the conversion of the $3^{\prime}$-protruding termini of SAGE concatemers into blunt ends represents an efficient and robust cloning strategy. The additional step of bluntending takes less than $1 \mathrm{~h}$ and requires only T4 DNA polymerase. No further purification step is required because the reaction mixture is directly used for ligation, and the protocol can be used for every vector containing an EcoRV/ Eco32I restriction endonuclease recognition site. This avoids problems associated with the often used pZERO vector (Invitrogen, Karlsruhe, Germany), which was sometimes shown to be unstable, and eliminates the need of the otherwise rarely used antibiotic zeomycin. In conclusion, the advantages mentioned above make our protocol a versatile, efficient, and inexpensive technique that should prove very helpful for the construction of SAGE libraries.

\section{REFERENCES}

1.Velculescu, V.E., L. Zhang, B. Vogelstein, and K.W. Kinzler. 1995. Serial analysis of gene expression. Science 270:484-487.

2.Jasper, H., V. Benes, A. Atzberger, S. Sauer, W. Ansorge, and D. Bohmann. 2002. A genomic switch at the transition from cell proliferation to terminal differentiation in the Drosophila eye. Dev. Cell 3:511-521.

3.Boon, K., E.C. Osorio, S.F. Greenhut, C.F. Schaefer, J. Shoemaker, K. Polyak, P.J. Morin, K.H. Buetow, et al. 2002. An anatomy of normal and malignant gene expression. Proc. Natl. Acad. Sci. USA 99:11287-11292.

4.Liang, P. 2002. SAGE Genie: a suite with panoramic view of gene expression. Proc. Natl. Acad. Sci. USA 99:11547-11548.

5.Saha, S., A.B. Sparks, C. Rago, V. Akmaev, C.J. Wang, B. Vogelstein, K.W. Kinzler, and V.E. Velculescu. 2002. Using the transcriptome to annotate the genome. Nat. Biotechnol. 20:508-512.

6.Datson, N.A., J. Perk-de Jong, M.P. van den Berg, E.R. de Kloet, and E. Vreugdenhil. 1999. MicroSAGE: a modified procedure for serial analysis of gene expression in limited amounts of tissue. Nucleic Acids Res. 27:1300-1307.

7.Angelastro, J.M., L.P Klimaschewski, and O.V. Vitolo. 2000. Improved NlaIII digestion of PAGE-purified $102 \mathrm{bp}$ ditags by addition of a single purification step in both the SAGE and microSAGE protocols. Nucleic Acids

Figure 1. Colony-PCR amplification of SAGE library inserts cloned by blunt-end ligation with Eco32I-cut vector. Colony-PCR was performed by using M13 forward and reverse primers in a $50-\mu \mathrm{L}$ Ten microliters of each reaction were analyzed in a $1 \%$, $60^{\circ} \mathrm{C}$ for $30 \mathrm{~s}$, and $72^{\circ} \mathrm{C}$ for $2 \mathrm{~min}$ for $35 \mathrm{cycles}$. fied concatemers of 300-500 bp (fraction I) in length. (B) Insert amplification of gel-purified concatemers of 500-1000 bp (fraction II) in length. Differences in the intensity of the insert bands were due to the applied technique (i.e., colony-PCR). The asterisk marks a colony-PCR in which two independent clones had been accidentally amplified; filled squares mark religated clones without an insert; filled triangles indicate clones where the PCR yielded no amplification product, probably because of missing primer binding site(s). 


\section{Benchmarks}

Res. 28:e62.

8.Lee, S., J.J. Chen, G.L. Zhou, and S.M. Wang. 2001. Generation of high-quantity and quality tag/ditag cDNAs for SAGE analysis. BioTechniques 31:348-354.

9.Peters, D.G., A.B. Kassam, H. Yonas, E.H O'Hare, R.E. Ferrell, and A.M. Brufsky. 1999. Comprehensive transcript analysis in small quantities of mRNA by SAGE-Lite. Nucleic Acids Res. 27:e39.

10.Powell, J. 1998. Enhanced concatemer cloning - a modification to the SAGE (serial analysis of gene expression) technique. $\mathrm{Nu}-$ cleic Acids Res. 26:3445-3446.

11.Kenzelmann, M. and K. Muehlemann. 1999. Substantially enhanced cloning efficiency of SAGE (serial analysis of gene expression) by adding a heating step to the original protocol. Nucleic Acids Res. 27:917-918.

12.Sambrook, J. and D.W. Russell. 2001. Molecular Cloning: A Laboratory Manual. CSH Laboratory Press, Cold Spring Harbor, NY.

13.Koesters, R. and M.V. Doeberitz. 2002. Im proved blunt-end cloning by replacing EcoRV with Eco32I. BioTechniques 32:1244-1246.

This research was supported by $D F G$ grant no. DFG No428/1-1 to H.G.N. and E.F. Address correspondence to Dr. H.G. Nothwang, Abt. Tierphysiologie, Universität Kaiserslautern, Postfach 3049, D67653 Kaiserslautern, Germany. e-mail: nothwang@rhrk.uni-kl.de

Received 18 December 2002; accepted 28 January 2003.

\section{Alexander Koehl, Eckhard Friauf, and Hans Gerd Nothwang \\ Universität Kaiserslautern Kaiserslautern, Germany}

\section{Improved Host Range Selection for Recombi- nant Modified Vaccinia Virus Ankara}

BioTechniques 34:694-700 (April 2003)

Modified vaccinia virus Ankara (MVA) is a valuable tool for the expression of recombinant genes and can be used for such different purposes as the in vitro study of protein functions or the in vivo induction of antigen-specific cellular or humoral immune responses. A major advantage of MVA is that it allows for high-level gene expression despite being replication defective in human cells and most mammalian cells. MVA as a vaccine has an excellent track record for safety, can be handled under biosafety level 1 conditions, and has proven to be immunogenic and protective when delivering heterologous antigens in animals (18 ), and first human candidate vaccines have proceeded into clinical trials (911). We previously developed an efficient method for the selection of recombinant MVA by transient host range gene expression (12), using the vaccinia virus $K 1 L$ gene function as a stringent marker to rescue MVA growth on rabbit kidney RK-13 cells.

When using this straightforward selection protocol for the construction of multiple recombinant MVA carrying heterologous gene sequences including the $g f p$ gene from the jellyfish $A e$ quorea victoria, we observed that part of the isolated recombinant MVA was able to grow on RK13 cells but did not express the target gene of interest. Upon molecular analysis of multiple isolated MVA, we detected two different failures: $(i)$ at the insertion site for the recombinant gene presence of only the K1L marker gene, no recombinant gene inserted and (ii) the region of natural deletion II within the MVA genome is affected/truncated (Figure 1A). The first observation is due to an initial single crossover event between the flank I gene sequences in the MVA genome and flank I repeat sequences of the transfer plasmid, followed by a second event of homologous recombination between the remaining flank I se- quences, which results in the stable insertion of only the K1L marker sequence into the MVA genome. The second observation occurs when homologous recombination events take place between the K1L gene sequences in MVA and the transfer plasmid. The possibility of the latter recombination event has also been suggested by Tscharke and Smith (13).

To test the respective importance of these undesired recombination events and to possibly improve the transient K1L selection technique, we adopted two measures: construction of MVA without K1L [MVA ( $\left.\mathrm{II}_{\text {new }}\right)$, Figure 1B] and the design of a new transfer plasmid (pIII $\Delta \mathrm{HR}$, Figure 1C). To delete the remaining 261-bp K1L sequence from the MVA genome, we constructed the deletion plasmid $\mathrm{pII}_{\text {new }} \mathrm{LZ}$-gpt-del: flank 1 of pUCII-LZ (14), a plasmid previously used for homologous recombination into the site of deletion II within the MVA genome, was substituted by a new flank (K2L) that did not contain the $K 1 L$ gene sequences and had been isolated by PCR from MVA genomic DNA using primers IIf1newA，5'-CAGCTGCAGCGGCCGCCTTACACCGTACCC-3', and IIf1newB, 5'-CAGGCATGCGTAGAACGTAGATCCGG-3'. Additionally, a 315-bp repeat of the $5^{\prime}$ region of the N2L ORF (also isolated by PCR, using primers delIIA, 5'-CAGCTGCAGCCATAATGGTCAATCGCC-3', and delIIB, 5'-CAGGCGGCCGCGGTATTCGATGATTATTTTTAACAAAATAAC-3') was inserted downstream of the LacZgpt-cassette, yielding pII $_{\text {new }}$ LZ-gpt-del and allowing for the deletion of the marker cassette upon homologous recombination of N2L gene sequences. MVA ( $\left.\mathrm{II}_{\text {new }}\right)$ was generated by the transfection of pII ${ }_{\text {new }}$ LZ-gpt-del DNA into primary chicken embryo fibroblasts (CEF) infected with MVA (clonal isolate F6), followed by the selection of $\beta$ galactosidase-producing viruses in the presence of mycophenolic acid, as previously described (7). After selection of recombinant MVA, selective pressure was removed, and marker-free viruses were isolated (Figure 1B). Additionally, to eliminate the possibility of homologous recombination between MVA genomic sequences and MVA repeat sequences in MVA transfer plasmids, we designed a new plasmid pIII $\Delta \mathrm{HR}$ that 